\title{
The influence of playing surface on injury risk in italian elite rugby players
}

\author{
Riccardo Maria Lanzetti ${ }^{1}$ \\ Domenico Lupariello ${ }^{1}$ \\ Teresa Venditto ${ }^{2}$ \\ Pierpaolo Rota ${ }^{1}$ \\ Matteo Guzzini' \\ Antonio Vadalà 1 \\ Attilio Rota ${ }^{3}$ \\ Andrea Ferretti ${ }^{1}$ \\ 1 Orthopaedic Unit and "Kirk Kilgour" Sports Injury \\ Centre, S. Andrea Hospital, "Sapienza" University \\ of Rome, Italy \\ 2 Department of Physical Medicine and Rehabilita- \\ tion. Board of Physical Medicine and Rehabilitation, \\ Department of Anatomy, Histology, Forensic \\ Medicine and Orthopedics, "Sapienza" University \\ of Rome, Italy \\ ${ }^{3}$ Ospedale Sandro Pertini, Roma, Italy
}

Corresponding author:

Domenico Lupariello

Orthopaedic Unit and "Kirk Kilgour" Sports Injury

Centre, S. Andrea Hospital

Via Grottarossa 1035-1039

00189 Rome, Italy

E-mail: domenico.lupariello@gmail.com

\section{Summary}

Background: There is a growing interest in the use of artificial turf surfaces in rugby. In particular, artificial surfaces may be an useful means of increasing participation in the sport by allowing greater usage of a given pitch, especially in regions where natural turf pitches are difficult to maintain.

Methods: The incidence of site, nature, cause, and severity of training and match injuries was prospectively recorded in two professional teams (one equipped with World Rugby certified third generation artificial turf and the other with natural grass over the 2014-2015 season).

Results: A total of 23,840 minutes of exposure was displayed for the whole sample, 1,440 minutes during matches and 22,400 during training sessions.

We recorded 37 (48\%) traumatic injuries and 39 $(52 \%)$ overuse injuries. For traumatic injuries, we did not find significant differences in the overall risk injury between grass and artificial turf considering match exposure and training sessions. For overuse injuries, there were significant differences in the overall risk injury between grass and artificial turf considering match exposure $(p=0.03)$ and training sessions $(p=0.02)$.

Conclusion: In elite Italian rugby players, artificial turf seems to be safe in regards to traumatic injury while it seems to be a risk factor for overuse injuries.

Level of evidence: II.

KEY WORDS: artificial turf, rugby, sports traumatology.

\section{Introduction}

Rugby is becoming one of the most popular worldwide contact sports. In 2015, the total number of registered players in the world increased from 2.56 million to 2.82 million while the total number of non-registered rugby players rose from 4.47 million to 4.91 million. More than one million children were also introduced to the sport during the year ${ }^{1}$.

Europe is the country with the largest number of participants ${ }^{1}(1,088,654$ registered players) and with the most ancient tradition. In fact, the first teams were founded at the end of the 1800s.

Over the past 15 years, there was a significant increase in the number of rugby players in Italy. Indeed, in 2015 there were 82,143 athletes and 810 teams registered. This was double of the number of players for the years before $2000^{1}$.

For this reason in the last few years there was a great interest in developing new technologies like artificial turfs to improve the game diffusion in different areas.

Albeit natural grass is the traditional playing surface for professional competitions and training, recently, World Rugby has focused on improving game quality by permitting the use of synthetic turf at all playing levels, ensuring a consistent playing surface that replicates the playing qualities of good quality natural grass $^{2}$.

Third generation synthetic turf comprises a stone base, a shock pad, carpet and rubber infill. Such surfaces are specifically designed to replicate more accurately the mechanical response of natural turf according to the World Rugby Regulation 22 introduced in $2003^{2}$. 
Moreover, in the last few years, there was a growing interest in the use of artificial turf surfaces in different sports with the increase in publications about the safety of these surfaces, in particular in European elite soccer leagues ${ }^{3-6}$. Despite this interest, only a few elite rugby teams have used artificial turf for their matches or training ${ }^{7}$. Indeed, there are few studies that analyse the safety of different playing surfaces in elite rugby championships ${ }^{8-11}$.

The aim of this study was to investigate the risk of acute and overuse injuries in elite rugby players in Italian major league on artificial turf compared with natural grass. Our hypothesis is that there are differences in the injury incidence on artificial turf and natural grass.

\section{Methods}

\section{Subjects (population)}

The incidence, site, nature, cause, and severity of training and match injuries was prospectively recorded in two professional teams (one equipped with World Rugby certified third generation artificial turf and the other with natural grass over the 2014-2015 season. All players enrolled were competing in the first division professional Italian national rugby League (Eccellenza Championship).

The following player details were completed: normal playing position, date of birth, height $(\mathrm{cm})$ and body mass $(\mathrm{kg})$.

Match exposures were calculated based on 15 players ( 8 forwards and 7 backs) being exposed for 80 minutes. Training exposure was calculated as the total number of players (forwards and backs) attending each training session multiplied by the number and length (minutes) of sessions. Each player participated in four organised field training sessions per week, with each training session lasting approximately 70 minutes.

\section{Data collection}

Baseline data were recorded at the start of the season for each player in two teams that trained and played matches on third generation artificial turf and natural grass. Medical history, particularly on previous ligament injuries, was also collected. Individual players' exposure to training sessions and competitions was registered. Data were collected by medical staff records in accordance with the King's model definition ${ }^{12}$.

The injury recording involved the match report forms completed after each competition. After the match and training session, the examiner collected the injury forms and imported them into a database.

No personal data were recorded on the injury forms or stored in the injury database and informed consent was obtained.

We did not include injuries or other medical conditions occurring outside Eccellenza Championship matches and training sessions.
The study protocol was approved by the hospital's Ethical Review Board and it was conducted in accordance with the principles of the Declaration of Helsinki and its amendments. Subjects were fully informed of the characteristics of the study and they gave their consent.

We conducted our research ethically according to international standards and as required by the Journal as described in Padulo et al. ${ }^{13}$.

\section{Definitions}

A recordable injury was defined as a sprain, strain, contusion, or concussion and as contact and noncontact. Injury was defined as "any physical complaint sustained by a player during a match or training that prevented the player from taking a full part in training or match play activities for one or more days beyond the day of injury"14.

Secondary outcomes included: i) injury severities that were reported as mean $\pm S D$ values (days), median, and inter quartile range (IQR) values (days), and grouped within severity categories as per the international consensus recommendations: minimal (2-3 days), mild (4-7 days), moderate (8-28 days), and severe (> 28 days); ii) injury mechanisms, that were differentiated into traumatic and overuse. Traumatic injuries are caused by an external event, while overuse injuries are caused by repeated micro-trauma without a single, identifiable event being responsible for the injury ${ }^{14}$.

\section{Statistical analysis}

The comparison of proportions (expressed as percentage) was performed by Chi-squared test according to Campbell I and Richardson JTE ${ }^{15}$.

The probability of developing injury within a specified period of time was calculated as incidence rate (IR) that was recorded as the number of injuries $/ 1,000$ hours per player of total exposure for each injury. Total exposure is athlete-time at risk and it was calculated as the total number of athletes (injured and uninjured) multiplied by their average participation time (expressed as the number of matches and training sessions). The matches and training sessions were added together and referred to as athlete-exposures, and the injury rates were expressed per 1,000 hours per player ${ }^{14}$. IR difference was used to compare the IRs between artificial turf and natural grass per 1,000 hours per player.

The Mann-Whitney test was used to compare artificial turf and natural grass according to injury severity.

In accordance with Hopkins et al. ${ }^{16}$, logistic regression was used to identify risk factors for injury using the following factors as explanatory variables: age, BMI, and artificial turf/natural grass.

The Statistical Package for social Sciences (SPSS) version 18 was used for calculations. All data were analysed by a single researcher. Computed $P$ values were 2 -sided, and $p<0.05$ was used to determine statistical significance. 


\section{Results}

Participants underwent 320 training sessions (each group was exposed to 160 training sessions of 70 minutes each) and 18 matches (each group was exposed to 9 matches of 80 minutes each). A total of 23,840 minutes of exposure was displayed for the whole sample, 1,440 minutes during matches and 22,400 during training sessions. Baseline characteristics of the sample are reported in Table I.

A total of 76 injuries were recorded, of which 52 $(68 \%)$ injuries on artificial turf and $24(32 \%)$ on natural grass (Difference $=36 \%, 95 \% \mathrm{Cl}: 57.2 \%$ to $97 \%$, Chi-squared $=8.55, p=0.00035$ ).

The IRs per 1,000 hours per player of exposure were 7.74 (95\% Cl: 5.78 to 10.16$)$ and 4.02 (95\% Cl: 1.02

Table I. Baseline characteristics of the sample.

\begin{tabular}{lcc}
\hline AGE & & \\
& Artificial & $26.40 \pm 3.08$ \\
& Natural & $25.14 \pm 4.95$ \\
BMI & & \\
& Artificial & $30.11 \pm 3.13$ \\
& Natural & $29.49 \pm 3.33$ \\
Matches & & \\
& Artificial & $\mathrm{N}=9,10.800^{*}$ \\
Training session & \\
& Natural & \\
Injury severity (days) & Natural & $\mathrm{N}=160,347.200^{*}$ \\
& Natifial & $\mathrm{N}=160,392.000^{*}$ \\
& Natural & $22.44 \pm 23.83$ \\
\hline
\end{tabular}

$\mathrm{N}=$ Number; * player-minutes. to 10.16) on artificial turf and natural grass, respectively. The rate ratio of injuries on artificial surfaces to grass surfaces was 1.92 (95\% Cl: 1.62-3.26). There was a significant difference in the overall risk injury between grass and artificial turf [IR difference $=3.72$ (95\% Cl: 1.02 to 6.42 ), $p=0.00069]$. According to match exposure, the IRs per 1,000 hours per player of exposure were $3.67(95 \% \mathrm{Cl}: 2.35$ to 5.46$)$ and 1.90 (95\% Cl: 0.94 to 3.40 ) on artificial turf and natural grass, respectively. The rate ratio of injuries on artificial surfaces to grass surfaces was $1.93(95 \% \mathrm{Cl}$ : $0.91-4.37)$. There was a significant difference in the overall risk injury between grass and artificial turf [IR difference $=1.77(95 \% \mathrm{Cl}$ : -0.11 to 3.66$), p=0.06$.

According to training sessions exposure, the IRs per 1,000 hours per player of exposure were 155.56 (95\% Cl: 103.37 to 224.82$)$ and 72.22 (95\% Cl: 38.45 to 123.502) on artificial turf and natural grass, respectively. The rate ratio of injuries on artificial surfaces to grass surfaces was $2.15(95 \% \mathrm{Cl}, 1.07-4.52)$. There was a significant difference in the overall risk injury between grass and artificial turf [IR difference $=83.33$ (95\% Cl: 13.61 to 153.05$), p=0.019]$.

Where injury severities are concerned, we did not find significant differences between artificial turf and natural grass $(p=0.35, Z=0.93)$ (Fig. 1).

For injury mechanisms, we recorded 37 (48\%) traumatic injuries (21 and 16 injuries on artificial turf and natural grass, respectively) and 39 (52\%) overuse injuries (31 and 9 injuries on artificial turf and natural grass, respectively), $p=0.62$. For traumatic injuries, we did not find significant differences in the overall risk injury between grass and artificial turf considering match exposure $(p=0.56)$ and training sessions $(p=0.65)$ (Tab. II). For overuse injuries, there were significant differences in the overall risk injury between grass and artificial turf considering match exposure $(p=0.03)$ and training sessions $(p=0.02)(T a b . I I)$.

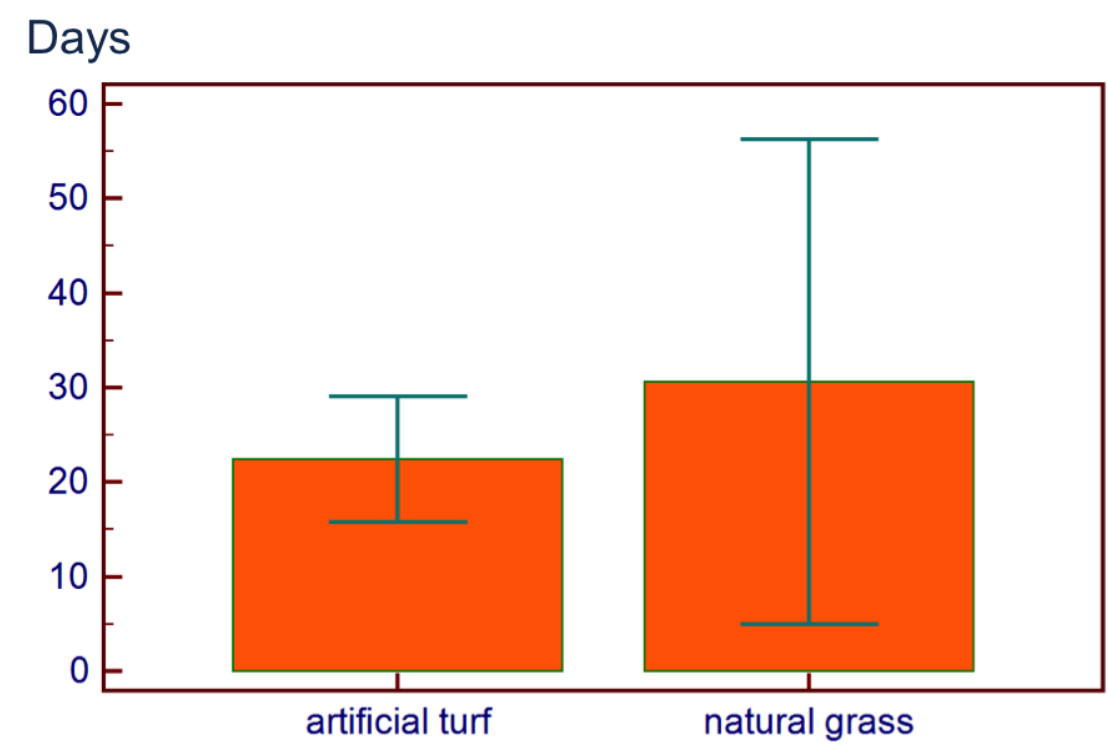

Figure 1. Comparison between artificial turf and natural grass according to time lost. 
Table II. Injuriy mechanism.

\begin{tabular}{|c|c|c|c|c|c|c|c|}
\hline & & \multicolumn{3}{|c|}{ Matches exposure } & \multicolumn{3}{|c|}{ Training sessions } \\
\hline & & $\mathbf{N}$ & IRs (CI 95\%) & RR (Cl 95\%) & $\mathbf{N}$ & IRs (Cl 95\%) & RR (Cl 95\%) \\
\hline \multicolumn{8}{|c|}{ Direct } \\
\hline - & $\begin{array}{l}\text { Artificial turf } \\
\text { Natural grass }\end{array}$ & $\begin{array}{l}15 \\
12\end{array}$ & $\begin{array}{l}83.33(46.64-137.44) \\
66.66(34.48-116.45)\end{array}$ & $1.25(0.54-2.92)$ & $\begin{array}{l}6 \\
4\end{array}$ & $\begin{array}{l}0.91(0.34-1.99) \\
0.69(0.18-1.76)\end{array}$ & $1.33(0.31-6.40)$ \\
\hline \multicolumn{8}{|c|}{ Indirect } \\
\hline $\begin{array}{l}- \\
-\end{array}$ & $\begin{array}{l}\text { Artificial turf } \\
\text { Natural grass }\end{array}$ & $\begin{array}{l}11 \\
2\end{array}$ & $\begin{array}{l}61.11(30.56-109.34) \\
11.11(1.34-40.14)\end{array}$ & $5.5(1.20-51.06)$ & $\begin{array}{l}20 \\
7\end{array}$ & $\begin{array}{l}3.06(1.86-4.72) \\
1.21(0.48-2.49)\end{array}$ & $2.53(1.03-7.08)$ \\
\hline
\end{tabular}

$\mathrm{N}=$ number; IRs=Incidence Rate; RR= Rate Ratio.

Finally, logistic regression analysis revealed that artificial turf/natural grass was associated with the occurrence of injury in rugby players $(p=0.0037)$.

\section{Discussion}

The most important finding of this study is an increased risk of overuse injury on artificial turf playing surfaces in Italian rugby elite players.

However, there are no significant differences in traumatic injury risk in matches or in training.

This is the first study to evaluate the risk of injury considering several risk factors (age, BMI and artificial turf/natural grass) as independent variables in a logistic regression analysis.

Analyzing the current literature: Fuller et al. ${ }^{9}$ conducted a two-season investigation comparing match injuries sustained on artificial turf and natural grass by six Rugby Union teams competing in the Hong Kong Division 1 and training injuries sustained by two teams in the English Premiership. The Authors reported no significant differences in the incidence of match and training injuries between the two surfaces, but the Authors did not analyse traumatic and overuse injuries separately.

Williams et al. ${ }^{8}$ investigated the influence of an artificial playing surface on injury risk and perceptions of muscle soreness in elite English Premiership Rugby Union players. Also, in this paper, the Authors reported no significant differences between surfaces.

However, the Authors showed that muscle soreness was consistently higher over the days following a match on artificial turf in comparison with a match played on natural grass.

Our results, according to current literature show that there were no significant differences in the overall risk of traumatic injury for rugby union played on artificial turf versus grass pitches.

This could be due to the fact that traumatic injuries, unlike the overuse injuries, are often caused by player-to-player contact, and are not dependent on the playing surface.
However the size of the sample population precluded obtaining strong statistical evidence about specific diagnoses such as ankle and knee ligament injuries, which have been identified in previous studies of other sports to have higher rates on artificial turf ${ }^{17-19}$. For this reason, further studies are needed to analyse specific injuries.

Instead, comparing our results to other sports with a larger study population, some differences were noticed. The risk of injury from playing soccer on artificial turf has been investigated in both the profession$\mathrm{al}^{3,17}$ and non-professional ${ }^{5,20}$ soccer games with no significant differences in the overall risk of injury identified in any of the studies, although some minor differences were observed for some specific injuries. However, comparing rugby and soccer would not be appropriate because of the different nature and features of these sports and the different influence of the playing surface.

In fact, comparing our data with a more comparable sport, the results are in accordance with those of previous studies on elite male American Football. Despite the fact that $\operatorname{Orchard}^{19}$, in some epidemiological studies of American Football, reported similar overall incidences of injury on artificial turf and grass, others identified specific injuries, such as knee and ankle ligament injuries, for which the incidence was higher on artificial turf.

In fact, different Authors reported that the overall rate of football injuries has been reported to be significantly higher on artificial surfaces compared with natural grass $^{21-25}$.

However, the strength of our study is to analyse the difference between traumatic and overuse injury risk on natural grass and artificial turf $(p=0.02)$.

In fact, the most interesting finding of our study is the presence of an increased risk of overuse injuries in artificial turf.

Although this finding, in our opinion, should be supported by further studies with a larger simple size, it can be justified by a different elasticity and adherence of the pitch that could lead to an increase in this type of injuries. 
In fact, overuse injuries are more likely to be linked to the playing surface due to the effects of the different traction and cushioning characteristics of the two surfaces $^{26}$. It has been speculated that foot-surface traction will impact on the translational and rotational forces transmitted through the lower-limb joints during cutting and turning activities and foot-surface cushioning effects may impact on the vertical forces transmitted through the lower-limb bones and soft tissues $^{27}$. It has also been suggested that differences in playing surface characteristics affect athletes' movements, which may impact on the incidence and severity of injury. Meyers and Barnhill ${ }^{18}$ even postulated that different playing surfaces had their own characteristic injury patterns.

\section{Limits}

Therefore, the type of grass on natural playing surfaces may affect the risk of injury, even if multiple risk factors should be considered to estimate the real incidence of athlete injuries. Indeed, the present study has some limitations: field moisture, precipitation, ambient temperature, player position, type of play, period in the game, timing of game within the season, and type of footwear have not been incorporated simultaneously to determine their interaction and significance.

Moreover, because of the size of the sample population, further surveillance is required before inferences regarding specific injury diagnoses can be made.

Despite these limitations, the strength of the present study is that it is based on the Ecological approach. In conclusion, in elite Italian rugby players, artificial turf seems to be safe in regards to traumatic injury while it seems to be a risk factor for overuse injuries. However continued surveillance is required to allow analyses of specific injury diagnoses and smaller overall differences in injury risk to be carried out.

\section{Conflict of interest}

The Author has no financial or personal relationships with other people or organizations that could inappropriately influence their work.

\section{References}

1. Player Numbers, 2015, http://www.worldrugby.org/development/player-numbers

2. IRB. Regulation 22. Standard relating to the use of artificial playing surfaces. Dublin. 2003.

3. Lanzetti RM, Ciompi A, Lupariello D, Guzzini M, De Carli A, Ferretti A. Safety of third-generation artificial turf in male elite professional soccer players in Italian major league. Scand $\mathrm{J}$ Med Sci Sports. 2016.

4. Dragoo JL, Braun $\mathrm{HJ}$, Harris $\mathrm{AH}$. The effect of playing surface on the incidence of ACL injuries in National Collegiate Athletic Association American Football. Knee. 2013;20(3):191-195.

5. Fuller CW, Dick RW, Corlette J, Schmalz R. Comparison of the incidence, nature and cause of injuries sustained on grass and new generation artificial turf by male and female football play- ers. Part 1: match injuries. Br J Sports Med. 2007a;41(Suppl. 1):i20-i26.

6. Fuller CW, Dick RW, Corlette J, Schmalz R. Comparison of the incidence, nature and cause of injuries sustained on grass and new generation artificial turf by male and female football players. Part 2: training injuries. Br J Sports Med. 2007b;41(Suppl. 1):i27-i32.

7. Swaminathan R, Williams JM, Jones MD, Theobald PS. A kinematic analysis of the spine during rugby scrummaging on natural and synthetic turfs. J Sports Sci. 2016;34(11):10581066.

8. Williams S, Trewartha G, Kemp SP, Michell R, Stokes KA. The influence of an artificial playing surface on injury risk and perceptions of muscle soreness in elite Rugby Union. Scand J Med Sci Sports. 2016;26(1):101-108.

9. Fuller CW, Clarke L, Molloy MG. Risk of injury associated with rugby union played on artificial turf. J Sports Sci. 2010;28(5): 563-570.

10. Granatelli G, Gabbett TJ, Briotti G, et al. Match analysis and temporal patterns of fatigue in rugby sevens. J Strength Cond Res. 2014;28(3):728-734.

11. Padulo J, Granatelli G, Ruscello B, D'Ottavio S. The place kick in rugby. J Sports Med Phys Fitness. 2013;53(3):224-231.

12. King DA, Gabbett TJ, Gissane C, Hodgson L. Epidemiological studies of injuries in rugby league: suggestions for definitions, data collection and reporting methods. J Sci Med Sport. 2009; 12(1):12-19.

13. Padulo J, Oliva F, Frizziero A, Maffulli N. Muscles, Ligaments and Tendons Journal - Basic principles and recommendations in clinical and field science research: 2016 update. MLTJ. 2016;6(1):1-5.

14. Fuller CW, Ekstrand J, Junge A, Andersen TE, Bahr R, Dvorak J, Hagglund M, McCrory P, Meeuwisse WH. Consensus statement on injury definitions and data collection procedures in studies of football (soccer) injuries. Br J Sports Med. 2006;40 (3):193-201.

15. Campbell I. 200: Chi-squared and Fisher-Irwin tests of two-bytwo tables with small sample recommendations. Statistics in Medicine 26:3661-3675; Richardson JTE, 2011: The analysis of $2 \times 2$ contingency tables - Yet again. Statistics in Medicine 30:890.

16. Hopkins WG, et al. Risk factors and risk statistics for sports injuries. Clinical Journal of Sport Medicine. 2007;17:3:208-210.

17. Ekstrand J, Timpka T, Hagglund M. Risk of injury in elite football played on artificial turf versus natural grass: a prospective two-cohort study. Br J Sports Med. 2006;40(12):975-980.

18. Meyers MC, Barnhill BS. Incidence, causes, and severity of high school football injuries on FieldTurf versus natural grass: a 5-year prospective study. Am J Sports Med. 2004;32(7): 1626-1638.

19. Orchard J. Is there a relationship between ground and climatic conditions and injuries in football? Sports Med. 2002;32 (7):419-432.

20. Steffen K, Andersen TE, Bahr R. Risk of injury on artificial turf and natural grass in young female football players. Br J Sports Med. 2007;41(Suppl 1):i33-i37.

21. Gorse K, Mickey CA, Bierhals A. Conditioning injuries associated with artificial turf in two preseason football training programs. J Athl Train. 1997;32(4):304-308.

22. Guskiewicz KM, Weaver NL, Padua DA, Garrett Jr WE. Epidemiology of concussion in collegiate and high school football players. Am J Sports Med. 2000;28(5):643-650.

23. Hagel BE, Fick GH, Meeuwisse WH. Injury risk in men's Canada West University football. Am J Epidemiol. 2003;157(9):825833.

24. Powell JW, Barber-Foss KD. Injury patterns in selected high school sports: a review of the 1995-1997 seasons. J Athl Train. 1999;34(3):277-284. 
25. Ramirez M, Schaffer KB, Shen H, Kashani S, Kraus JF. Injuries to high school football athletes in California. Am J Sports Med. 2006;34(7):1147-1158.

26. Nigg B M. The validity and relevance of tests used for the assessment of sports surfaces. Medicine and Science in Sports and Exercise. 1990;22:131-139.

27. Kaila R. Influence of modern studded and bladed soccer boots and sidestep cutting on knee loading during match play conditions. American Journal of Sports Medicine. 2007; 35:15281536. 